OPEN ACCESS

Edited by:

Jonathan Shaw,

University of Sheffield,

United Kingdom

Reviewed by:

Mark Radosevich,

University of Tennessee,

United States

John Stolz,

Duquesne University, United States

${ }^{*}$ Correspondence:

Lukasz Drewniak

Idrewniak@biol.uw.edu.pl

Specialty section

This article was submitted to Microbiotechnology, Ecotoxicology

and Bioremediation,

a section of the journal

Frontiers in Microbiology

Received: 01 March 2017 Accepted: 09 May 2017

Published: 29 May 2017

Citation:

Uhrynowski W, Decewicz P,

Dziewit L, Radlinska M,

Krawczyk PS, Lipinski L, Adamska D

and Drewniak L (2017) Analysis of the

Genome and Mobilome of a

Dissimilatory Arsenate Reducing

Aeromonas sp. O23A Reveals

Multiple Mechanisms for Heavy Metal

Resistance and Metabolism.

Front. Microbiol. 8:936.

doi: 10.3389/fmich.2017.00936

\section{Analysis of the Genome and Mobilome of a Dissimilatory Arsenate Reducing Aeromonas sp. O23A Reveals Multiple Mechanisms for Heavy Metal Resistance and Metabolism}

\author{
Witold Uhrynowski', Przemyslaw Decewicz'2, Lukasz Dziewit², Monika Radlinska ${ }^{3}$, \\ Pawel S. Krawczyk ${ }^{4}$, Leszek Lipinski ${ }^{4}$, Dorota Adamska ${ }^{4}$ and Lukasz Drewniak ${ }^{1 *}$ \\ ' Laboratory of Environmental Pollution Analysis, Faculty of Biology, University of Warsaw, Warsaw, Poland, ${ }^{2}$ Department of \\ Bacterial Genetics, Institute of Microbiology, Faculty of Biology, University of Warsaw, Warsaw, Poland, ${ }^{3}$ Department of \\ Virology, Institute of Microbiology, Faculty of Biology, University of Warsaw, Warsaw, Poland, ${ }^{4}$ Institute of Biochemistry and \\ Biophysics, Polish Academy of Sciences, Warsaw, Poland
}

Aeromonas spp. are among the most ubiquitous microorganisms, as they have been isolated from different environmental niches including waters, soil, as well as wounds and digestive tracts of poikilothermic animals and humans. Although much attention has been paid to the pathogenicity of Aeromonads, the role of these bacteria in environmentally important processes, such as transformation of heavy metals, remains to be discovered. Therefore, the aim of this study was a detailed genomic characterization of Aeromonas sp. O23A, the first representative of this genus capable of dissimilatory arsenate reduction. The strain was isolated from microbial mats from the Zloty Stok mine (SW Poland), an environment strongly contaminated with arsenic. Previous physiological studies indicated that O23A may be involved in both mobilization and immobilization of this metalloid in the environment. To discover the molecular basis of the mechanisms behind the observed abilities, the genome of O23A ( 5.0 Mbp) was sequenced and annotated, and genes for arsenic respiration, heavy metal resistance (hmr) and other phenotypic traits, including siderophore production, were identified. The functionality of the indicated gene modules was assessed in a series of minimal inhibitory concentration analyses for various metals and metalloids, as well as mineral dissolution experiments. Interestingly, comparative analyses revealed that O23A is related to a fish pathogen Aeromonas salmonicida subsp. salmonicida A449 which, however, does not carry genes for arsenic respiration. This indicates that the dissimilatory arsenate reduction ability may have been lost during genome reduction in pathogenic strains, or acquired through horizontal gene transfer. Therefore, particular emphasis was placed upon the mobilome of O23A, consisting of four plasmids, a phage, and numerous transposable elements, which may play a role in the dissemination of $h m r$ and arsenic metabolism genes in the environment. The obtained results indicate that Aeromonas $\mathrm{sp}$. O23A is well-adapted to the extreme environmental conditions occurring in the 
Zloty Stok mine. The analysis of genome encoded traits allowed for a better understanding of the mechanisms of adaptation of the strain, also with respect to its presumable role in colonization and remediation of arsenic-contaminated waters, which may never have been discovered based on physiological analyses alone.

Keywords: Aeromonas spp., dissimilatory arsenate reduction, heavy metals resistance, genome, mobilome, plasmid

\section{INTRODUCTION}

Aeromonads are ubiquitous microorganisms found in different environmental niches such as fresh and marine waters, sediments and soil (e.g., Dumontet et al., 2000). Moreover, some Aeromonas spp. strains, recognized as opportunistic pathogens, have been isolated from wounds and digestive tracts of poikilothermic animals and mammals, including humans (e.g., Chen et al., 2016; Teunis and Figueras, 2016). Due to health threats related to the infections with these bacteria, many studies focus on determining the antibiotic resistance phenotypes and virulence factors of various Aeromonas strains (Rasmussen-Ivey et al., 2016). Much less attention is paid to thorough physiological and genomic analysis of Aeromonas spp. that have not been described as pathogenic, but which may be involved in processes beneficial from the environmental point of view, e.g., bioremediation and detoxification (Ogugbue and Sawidis, 2011).

Environmental isolates of Aeromonas spp. are known to affect the cycle of inorganic and organic matter in the environment (e.g., Wang et al., 2009; Watts and Lloyd, 2013). This is also the case for arsenic, a metalloid, which constitutes a particular threat to living organisms due to its high toxicity, but to which many microorganisms, including Aeromonads, evolved resistance (Drewniak et al., 2010). Much less common among Aeromonas spp. is the ability to directly utilize arsenic species to produce energy, for which dissimilatory arsenate reducing bacteria (DARB) are particularly known (Drewniak et al., 2015). This group of bacteria comprises a number of species belonging to different genera, i.a., Alkaliphilus, Bacillus, and Shewanella. Surprisingly, given the ubiquitousness and versatility of metabolism of Aeromonas spp., only two dissimilatory arsenate reducing strains belonging to this genus have so far been described: O23A (Drewniak et al., 2010) and OM4 (Drewniak et al., 2014). Both the strains originate from an ancient gold mine in Zloty Stok (SW Poland).

Aeromonas sp. O23A, the first known dissimilatory arsenate reducing Aeromonad, was isolated from bottom sediments and microbial mats found in arsenic-contaminated $(\sim 20,000 \mu \mathrm{g}$ As/l) effluents from the mine. Preliminary analyses carried out by Drewniak et al. (2010) showed that the strain is capable of arsenate respiration using lactate as the donor of electrons. Further, thorough investigation of the O23A physiology was carried out by Uhrynowski et al. (2017). The study revealed that $\mathrm{O} 23 \mathrm{~A}$ has a versatile metabolism, as it is capable of both aerobic and anaerobic growth using various electron donors and acceptors. Moreover, the strain shows high tolerance to extreme environmental conditions, such as contamination with heavy metals, both low and high $\mathrm{pH}$
(4-11) and temperature $\left(4-42^{\circ} \mathrm{C}\right)$. Furthermore, under anaerobic conditions, $\mathrm{O} 23 \mathrm{~A}$ may be involved in direct release of arsenic from rocks containing As-minerals. Interestingly, physiological studies indicated that $\mathrm{O} 23 \mathrm{~A}$ may facilitate not only mobilization, but also immobilization of arsenic in the environment. This trait is considered unique among DARB, as they typically promote the release of this metalloid from minerals, and thus increase the level of pollution (Okibe et al., 2014; Drewniak et al., 2015). Moreover, metagenomic analysis showed that O23A is one of the dominant DARB strains within the microbial mats community in the Zloty Stok gold mine (Drewniak et al., 2012). The mats may act as effective filters retaining heavy metal ions and other pollutants flowing through the mine waters (Drewniak et al., 2016), leading to their selfpurification.

The results of the physiological studies of Aeromonas sp. O23A confirm the key role of the strain in colonization and remediation of arsenic-contaminated waters. However, the genetic basis for all the processes in which $\mathrm{O} 23 \mathrm{~A}$ is involved is still unknown. Genomic studies may provide information on the already known capabilities of the strain, as well as those which have not yet been discovered based on the physiological analyses. For example, comparison with the fairly well characterized clinical isolates of Aeromonas spp. may be crucial in the assessment of risks associated with the potential presence of pathogenicity determinants related to, e.g., biofilm formation (Du et al., 2016) and siderophore production (Tomás, 2012). This may prove important in the application for the Generally Recognized As Safe (GRAS) status, lack of which may hinder the actual use of the strain in bioremediation, given its taxonomic relatedness to opportunistic pathogens. Comparative genomic analyses can also provide information about the direction of evolution of Aeromonas spp. genomes.

Therefore, the aim of this study was a detailed genomic characterization of Aeromonas sp. O23A, supported by physiological analyses, which allowed for: (i) an insight into the adaptation strategies of the strain to extreme environmental conditions, (ii) better understanding of its function within the microbial community and (iii) determination of its potential gene transfer mechanisms and relatedness of various Aeromonads. Genomic analyses were focused on chromosomal and mobilomeencoded heavy metal resistance and metabolism genes, while the physiological analysis was aimed to explore the range of heavy metal resistance and mobilization capabilities. This work, therefore, provides a novel insight into the abilities of Aeromonas sp. O23A, identifying and underlining the genetic basis of the biogeochemical changes observed in the environment in which the strain may potentially be involved. 


\section{MATERIALS AND METHODS}

\section{Media and Growth Conditions}

The investigated Aeromonas sp. O23A strain was isolated from waters, rock biofilms and bottom sediments from the Zloty Stok gold mine (SW Poland) by Drewniak et al. (2010). The strain was routinely grown under aerobic conditions in LB (Sambrook and Russell, 2001) or minimal salt (MS) medium (Drewniak et al., 2015) supplemented with $5 \mathrm{mM}$ lactate and yeast extract $(0.04 \% \mathrm{w} / \mathrm{v})$ as a source of vitamins, at the temperature of $26 \pm 2{ }^{\circ} \mathrm{C}$. Optimal growth conditions were determined previously by Uhrynowski et al. (2017).

\section{DNA Manipulations DNA Isolation}

Standard DNA manipulation methods were performed as described by Sambrook and Russell (2001). Total DNA was extracted using a kit (Genomic Mini, A\&A Biotechnology) from bacterial cells harvested by centrifugation of an overnight culture carried out in LB medium. Purified DNA was used as the template for genome sequencing. Plasmid DNA was isolated analogously using a kit (Plasmid Mini, A\&A Biotechnology) or by $\mathrm{CsCl}$ gradient method (Sambrook and Russell, 2001).

\section{Genome Sequencing, Assembly and Analysis}

Genome assembly was performed using a combination of Illumina short data reads and PacBio RS long reads. Two pairend libraries with an average insert size of 160 and 460 bp were prepared using the Illumina TruSeq v2 kit and sequenced on an Illumina HiScan with $2 \times 100 \mathrm{nt}$ read length. Illumina sequencing yielded 0.561 and 0.89 million reads, respectively, which were first trimmed to remove adaptor sequences using cutadapt (v1.8) and then quality trimmed using sickle2 (mean quality 30 , min. length $50 \mathrm{nt}$ ). PacBio sequencing data was obtained from the High-Throughput Sequencing Facility at the University of North Carolina (Chapel Hill, United States). Library sequencing with an average insert size of $20 \mathrm{~kb}$ resulted in 467,925 reads with the mean length of $1.48 \mathrm{~kb}$ (longest read: $23.14 \mathrm{~kb}$ ). Assembly of the obtained reads was performed using SPAdes (v.3.0.0) - careful option with PacBio reads supplied as filtered subreads fasta. This yielded 240 scaffold sequences, with a mean length of $20 \mathrm{~kb}$ (longest scaffold - $841.97 \mathrm{~kb}$ ). The scaffold assembly was verified using sequence reads obtained after PCR amplification of selected genome regions, as well as from the fosmid genome library of $\mathrm{O} 23 \mathrm{~A}$ created in pNGS FOS (Lucigen), which were sequenced in the DNA Sequencing and Oligonucleotide Synthesis Laboratory (oligo.pl) at the Institute of Biochemistry and Biophysics, Polish Academy of Sciences.

The obtained O23A chromosome sequence was annotated using Prokka 4 (v 1.10) and RAST (Aziz et al., 2008) and deposited in the GenBank database. Automatic annotation results for selected open reading frames (ORFs) were verified manually using Artemis software (Carver et al., 2008) and BLAST programs (Altschul et al., 1997) provided on the National Center for Biotechnology Information (NCBI) website ${ }^{1}$. Metabolic pathways

${ }^{1}$ http://blast.ncbi.nlm.nih.gov/Blast.cgi were assigned using Pathologic5 from the Pathway Tools 18.5 (Karp et al., 2011) ran on the Prokka output.

\section{Plasmid Annotation and Analysis}

Plasmid nucleotide sequences were analyzed using Clone Manager (Sci-Ed8) and Artemis software. Similarity searches were performed using the BLAST programs. The manually annotated sequences of $\mathrm{O} 23 \mathrm{~A}$ plasmids $\mathrm{p} 1-\mathrm{p} 4$ were deposited in the GenBank database.

\section{Phage Induction and Analysis}

Prophage sequences within the draft genome were identified using PHAge Search Tool (PHAST) (Zhou et al., 2011)2. To induce potential prophages in Aeromonas sp. O23A, bacterial cells were treated with mitomycin C, a classical inducer of lambdoid prophages. The resulting lysate was purified by PEG precipitation and $\mathrm{CsCl}$ density gradient separation. The visible band was collected and analyzed for the presence of phage particles by transmission electron microscopy (TEM). Genomic DNA of the phage was extracted from CsCl-purified viral particles and was subjected to high throughput resequencing. The obtained phage sequence was automatically annotated using the RAST server and the resulting annotations were then thoroughly manually curated. BLASTP and Psi-BLAST algorithms were used for the similarity searches in the $\mathrm{NCBI}^{3}$, UniProt ${ }^{4}$ and REBASE (Roberts et al., 2015) databases.

\section{Heavy Metal Resistance Analyses}

Heavy metal resistance capabilities of the strain were analyzed by determining the minimal inhibitory concentrations (MICs) of metal ions that completely inhibited the strain's growth. Cultures were carried out aerobically in titration plates, in LB medium amended with appropriately diluted stock solutions of analytical grade heavy metal salts $\left(3 \mathrm{CdSO}_{4} \times 8 \mathrm{H}_{2} \mathrm{O} ; \mathrm{CoSO}_{4} \times 7 \mathrm{H}_{2} \mathrm{O}\right.$; $\left.\mathrm{HgCl}_{2} ; \mathrm{K}_{2} \mathrm{Cr}_{2} \mathrm{O}_{7}\right)$. The growth of the strain was assessed by measuring the changes in the optical density at $600 \mathrm{~nm}$ $\left(\mathrm{OD}_{600 \mathrm{~nm}}\right)$ of the cultures compared to the non-inoculated controls using an automated plate reader (Sunrise, TECAN). Spectrophotometric measurements were carried out at 24-h intervals for 3 days, and changes in $\mathrm{OD}_{600 \mathrm{~nm}}>0.2$ throughout the experiment were considered positive results. All experiments were repeated three times. O23A was considered resistant to a given metal if it was capable of growth in the presence of at least: (i) $10 \mathrm{mM}$ of $\mathrm{As}(\mathrm{V})$, (ii) $1 \mathrm{mM}$ of $\mathrm{As}(\mathrm{III}), \mathrm{Cd}(\mathrm{II}), \mathrm{Co}(\mathrm{II}), \mathrm{Cr}(\mathrm{VI})$, $\mathrm{Cu}(\mathrm{II}), \mathrm{Ni}$ (II) or $\mathrm{Zn}(\mathrm{II})$, or (iii) $0.1 \mathrm{mM}$ of $\mathrm{Hg}$ (II) (Dziewit et al., 2013). MICs for $\mathrm{As}(\mathrm{III}), \mathrm{As}(\mathrm{V}), \mathrm{Cu}(\mathrm{II}), \mathrm{Ni}(\mathrm{II})$, and $\mathrm{Zn}(\mathrm{II})$ were determined in the previous study (Uhrynowski et al., 2017).

\section{Siderophore Production and Mineral Dissolution Analyses}

Aeromonas sp. O23A cultures for the studies of secondary metabolite production were carried out aerobically on GASN medium (Bultreys and Gheysen, 2000) for $72 \mathrm{~h}$ at $26^{\circ} \mathrm{C}$ as

\footnotetext{
${ }^{2}$ http://phast.wishartlab.com/

${ }^{3}$ http://www.ncbi.nlm.nih.gov

${ }^{4}$ http://www.uniprot.org/
} 
described by (Uhrynowski et al., 2017). Uninoculated medium was used as the control. The cultures were centrifuged and the obtained supernatants were passed through sterile $0.2 \mu \mathrm{m}$ filters, and used for mineral dissolution experiments. The experiments were carried out in $50 \mathrm{ml}$ tubes containing $40 \mathrm{ml}$ of culture supernatants with the addition of $10 \mathrm{~g} / \mathrm{l}$ of arsenopyrite or pyrite (approximately 1-2 $\mathrm{mm}$ fraction). The minerals were washed with $\mathrm{HCl}$ and distilled water to remove the oxidized surface and loose particles, dried, weighed, and sterilized. Samples were collected at the beginning of the experiments, and after 7 days of incubation with vigorous shaking. Iron concentrations were quantitatively analyzed by flame atomic absorption spectrometry (FAAS, AA Solaar M6 Spectrometer, TJA Solutions, Cambridge, United Kingdom), using a set of standard solutions (Merck, Darmstadt, Germany) prepared in $0.5 \mathrm{M} \mathrm{HNO}_{3}$. All experimental variants were carried out in duplicate.

\section{Phylogenetic Analysis}

The phylogenetic relationship between 30 validly assigned representatives of the genus Aeromonas, whose genomes have been sequenced, was analyzed based on the $r p o B$ gene nucleotide sequence similarity. The sequences were aligned using ClustalW software ${ }^{5}$. The obtained alignment was adjusted manually and then used to render a phylogenetic tree. The rooted tree was constructed using the distance matrix calculated by the NeighborJoining method (Saitou and Nei, 1987). The evolutionary distances were computed using the Kimura 2-parameter formula (Kimura, 1980). The bootstrap consensus tree was inferred from 1000 replicates (Felsenstein, 1985). All the above evolutionary analyses were conducted using MEGA7 software (Kumar et al., 2016).

\section{Nucleotide Sequence Accession Numbers}

The nucleotide sequences of Aeromonas sp. O23A chromosome and extrachromosomal replicons pO23A_p1-p4 have been annotated and deposited in the GenBank database (BioSample: SAMN06812489). The .gbk files may be found in the Supplementary Materials (Supplementary File S1).

\section{RESULTS AND DISCUSSION}

\section{General Features of the Aeromonas sp. O23A Genome}

The genome of Aeromonas sp. O23A is composed of a single circular chromosome of 4,811,606 bp and four circular plasmids: pO23A_P1 (4161 bp), pO23A_P2 (7621 bp), pO23A_P3 $(51,944 \mathrm{bp})$, and pO23A_P4 $(60,010 \mathrm{bp})$. The total size of the genome is $4,935,342 \mathrm{bp}$. The overall GC content of the chromosome is $58.4 \%$, which is consistent with the other sequenced Aeromonas genomes (whose GC content ranges between $58.17 \%$ (A. salmonicida subsp. salmonicida A449) and 62\% (A. hydrophila 4AK4) (Adamczuk and Dziewit, 2016).

\footnotetext{
${ }^{5}$ www.ebi.ac.uk/clustalw
}

The GC contents of the O23A plasmids ranges between $44.7 \%$ (pO23A_P3) and 54.3\% (pO23A_P1) (Figure 1 and Table 1). Interestingly, the GC contents of all the plasmids are significantly lower than the corresponding value for the chromosome. This phenomenon was also previously noticed in several metaanalyses of plasmids (e.g., Dziewit and Bartosik, 2014), and may be a consequence of the fact that bacteria cannot stably maintain horizontally acquired DNAs, whose GC content is higher than that of the chromosome (Nishida, 2013).

Automatic annotation using the RAST server identified 4710 genes (4535 within the chromosome and 162 within the plasmids) with an average length of $905 \mathrm{bp}$. The smallest distinguished gene (93 bp), encoding a hypothetical protein, was identified within the plasmid pO23A_P3, and the largest one (14,070 bp) was found within the chromosome and encoded a putative acetyltransferase. Additionally, 118 tRNA genes and 9 clusters of 16S-23S-5S rRNA (plus one additional 5S rRNA-encoding gene) were identified within the Aeromonas sp. O23A chromosome (Table 1).

A total of $3933(83.5 \%)$ predicted Aeromonas sp. O23A proteins were functionally categorized, which allowed for the calculation of the proportions in each COG category (Supplementary Table S1). Over $32 \%$ of the proteins with assigned COG numbers were described as involved in the overall cellular metabolism (i.e., categories C, G, E, F, H, and I). Interestingly, as many as 218 of the predicted proteins were classified into the COG category $\mathrm{P}$, which means that they are most probably involved in inorganic ion transport and metabolism (Supplementary Table S1). Moreover, the EC numbers were assigned to over 1000 of the predicted Aeromonas sp. O23A proteins. Most of them were classified as transferases (367), then oxidoreductases and hydrolases (212 each), lyases (109), ligases (77), and isomerases (73).

\section{Phylogeny of Aeromonas sp. O23A}

Phylogenetic analysis of $r p o B$ genes, revealed that Aeromonas sp. $\mathrm{O} 23 \mathrm{~A}$ is most closely related to Aeromonas salmonicida subsp. salmonicida A449 (Figure 2). This is in accordance with the previous phylogenetic analysis based on the 16S rRNA gene sequence comparison of $\mathrm{O} 23 \mathrm{~A}$ with other Aeromonads (Uhrynowski et al., 2017). The DNA sequences of this gene for these two strains are in 99\% identical. Moreover, in the bootstrap test, the strains were clustered together as the closest relatives in all the repeats, which corresponds to very high similarity of the sequences and indicates close phylogenetic relationship between the $\mathrm{O} 23 \mathrm{~A}$ and $\mathrm{A} 449$ strains.

This result may provide information about the origin of O23A. Potentially, the common ancestor of the two strains was brought to the mine waters by salmonid-type fish, which are typical hosts of $A$. salmonicida. The common evolutionary pathway may, however, indicate that $\mathrm{O} 23 \mathrm{~A}$ is potentially capable of infecting poikilothermic animals, making it unsuitable for use in open bioremediation systems before the genes for potentially dangerous traits are effectively silenced or deleted, (e.g., Darmon and Leach, 2014).

Alternatively, the common ancestor of O23A and A449 might not have been a pathogenic strain, and it became one as a result 


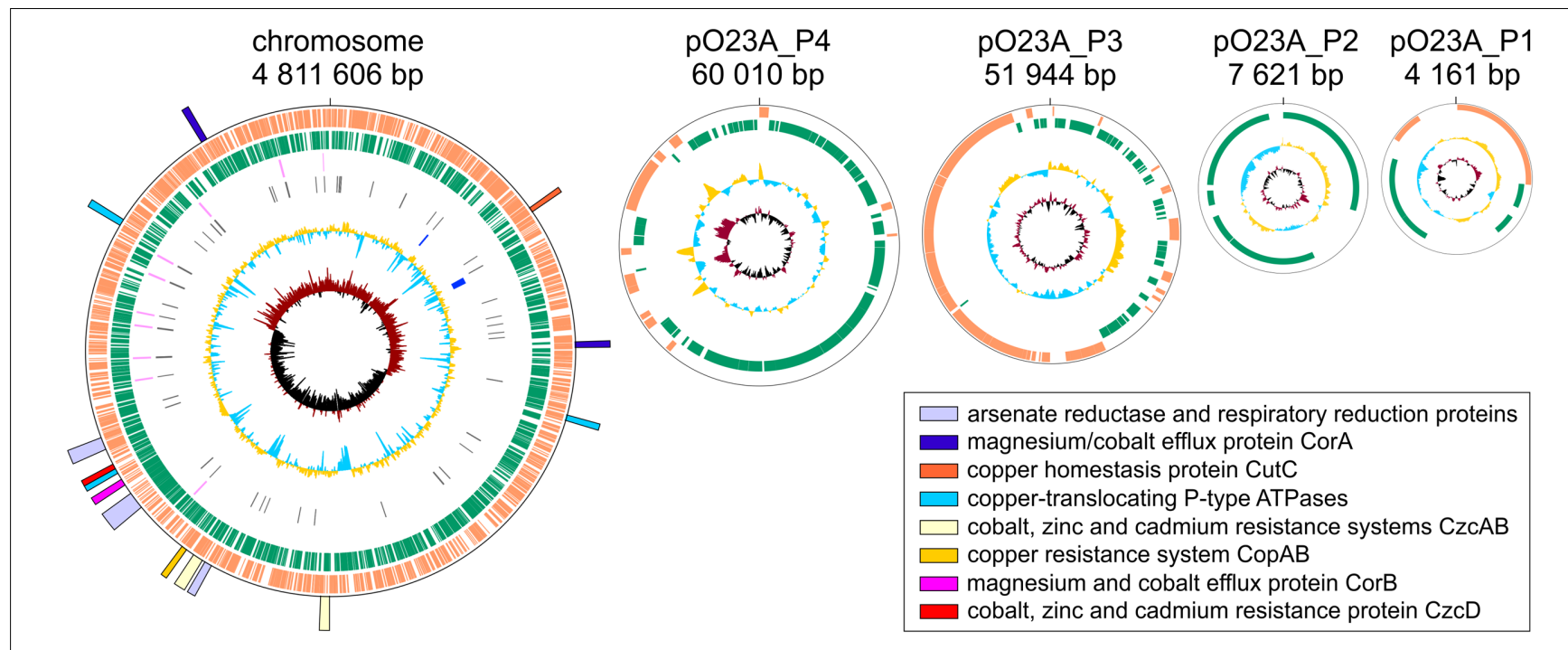

FIGURE 1 | Physical maps of the chromosome and plasmids of Aeromonas sp. O23A. Open reading frames are represented as orange and green blocks. The innermost circle represents GC skew, whereas the lager circles - GC content, CRISPR regions and RNA genes, respectively. The identified heavy metal resistance and metabolism modules are indicated as colored rectangles, forming the outermost part of the physical map of the O23A chromosome.

TABLE 1 | General features of the Aeromonas sp. O23A genome.

\begin{tabular}{|c|c|c|c|c|c|}
\hline General features & Chromosome & p023A_P1 & pO23A_P2 & pO23A_P3 & pO23A_P4 \\
\hline Size (bp) & $4,811,606$ & 4161 & 7621 & 51,944 & 60,010 \\
\hline GC content (\%) & 58.4 & 54.3 & 52.6 & 44.7 & 50.5 \\
\hline Coding density (\%) & 87.5 & 60.8 & 79.9 & 82.5 & 82.6 \\
\hline Number of genes & 4535 & 7 & 10 & 69 & 76 \\
\hline Number of tRNA genes & 118 & 0 & 0 & 0 & 0 \\
\hline $\begin{array}{l}\text { Number of } 16 S-23 S-5 S \\
\text { rRNA gene clusters }\end{array}$ & $\begin{array}{l}9 \text { (plus one additional } \\
\text { gene for } 5 \mathrm{~S} \text { rRNA) }\end{array}$ & 0 & 0 & 0 & 0 \\
\hline Phage regions & 1 & 0 & 0 & 0 & 0 \\
\hline
\end{tabular}

of gene transfer events between bacteria. This is probable, as the concentration of arsenic in these waters is high and may be toxic to aquatic organisms, preventing their access to the mine. In such a case, O23A may be incapable of infecting fish hosts. The above hypotheses however, have to be confirmed by in vivo studies.

\section{Mobilome Description}

To determine the role of horizontal gene transfer in shaping of the Aeromonas sp. O23A genome a thorough investigation of the distinguished mobile elements was performed. The mobilome of $\mathrm{O} 23 \mathrm{~A}$ is quite diversified, as the strain was found to carry four plasmids, one prophage and several putative transposable elements. All of these may play a crucial role in the dissemination of genes in the environment, and are discussed in detail below.

\section{Plasmids of O23A}

The plasmids of Aeromonas sp. O23A range in size from $\sim 4 \mathrm{~kb}$ to over $60 \mathrm{~kb}$. The smallest plasmid (pO23A_p1) comprises seven ORFs, and its backbone consists mainly of a putative RepB protein. The plasmid also encodes two potential transcriptional regulators and six hypothetical proteins of an unknown function.
Plasmid pO23A_p2 ( 7.5 kb) comprises 10 ORFs, including a potential DNA methylase and type II restriction enzyme, with CAGCTG specificity (PvuII). Based on amino-acid sequence comparisons the putative protein is almost identical with the restriction-modification system of $A$. hydrophila SNUFPC-A8. Interestingly, pO23A_p2 carries genes for a membrane protein of the type VI secretion system (T6SS), which may be a potential virulence determinant. Moreover, like all the plasmids of Aeromonas sp. O23A, except for pO23A_p1, pO23A_p2 contains a set of genes encoding proteins responsible for mobilization for conjugal transfer (MOB module).

The second-largest plasmid, pO23A_p3 ( 52 kb), apart from the replication protein, contains a partitioning system (ParA, ORF62) and a potential conjugal transfer module. In the case of pO23A_p3, the TRA module may be impaired or otherwise inactive, as the MOB system was also found. The potential function of many predicted proteins encoded by this plasmid remains unknown.

The backbone of the largest plasmid, pO23A_p4 ( 60 kb), consists of two potential replication proteins $\operatorname{RepA}$ and $\operatorname{RepB}$, as well as a complete set of transconjugal transfer genes (TRA module). Apart from the TRA module, this plasmid also carries 


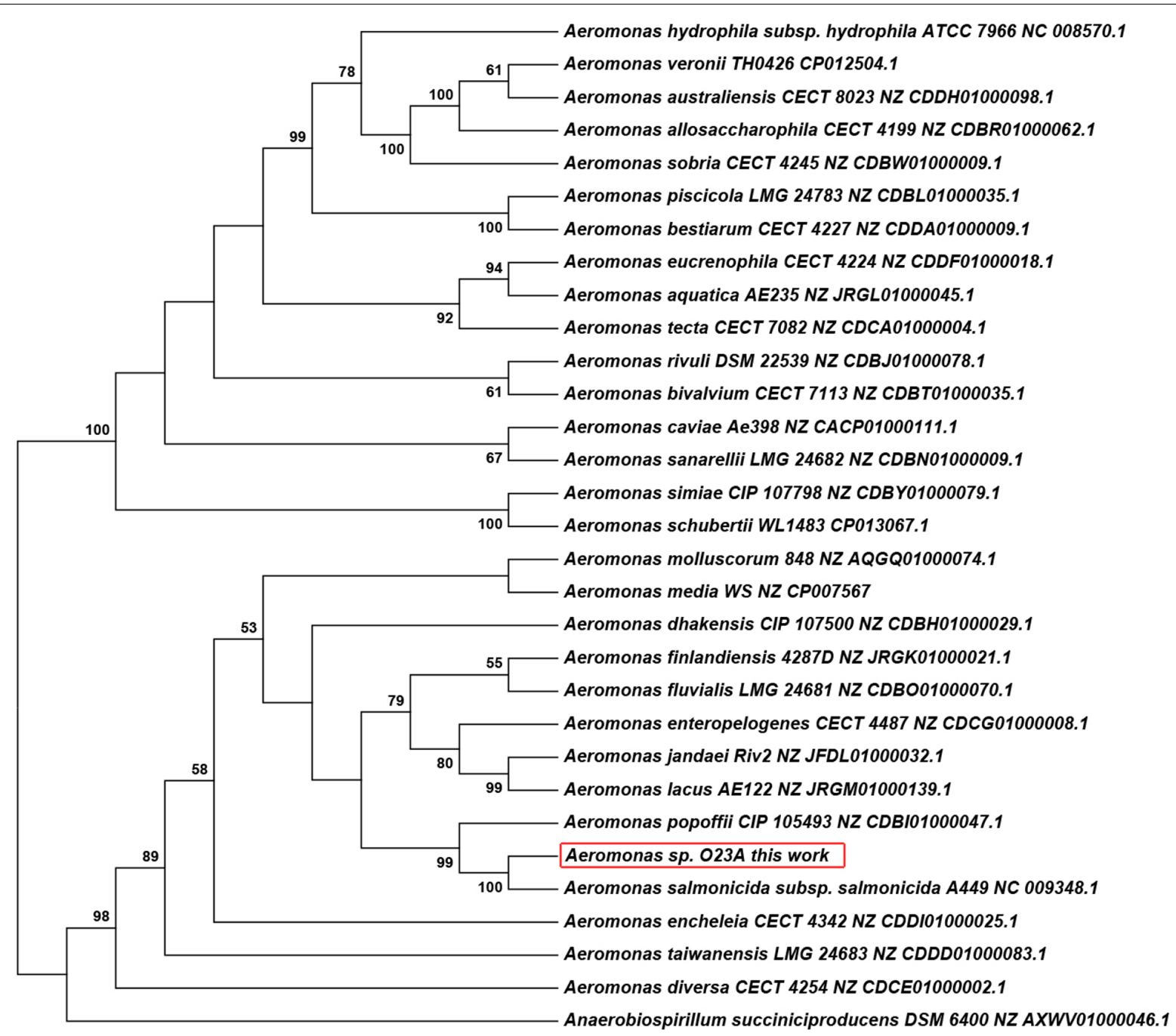

FIGURE 2 | Phylogenetic tree of Aeromonas spp. based on the rpoB genes sequences of $\mathbf{3 0}$ validly assigned representatives of this genus. The strain $\mathrm{O} 23 \mathrm{~A}$ analyzed in this work was indicated with a red frame. The rpoB gene sequence of Anaerobiospirillum succiniciproducens was used as the root. Accession numbers of the gene sequences used for the phylogenetic analysis are shown. The percentage of replicate trees, in which the associated taxa clustered together in the bootstrap test (1000 replicates) are shown next to the branches. Only values $>50 \%$ are shown.

a potential toxin-antitoxin module $\mathrm{HicA} / \mathrm{HicB}$ (ORF18 and ORF17, respectively). Among the phenotypic modules encoded by the plasmid putative tellurium resistance proteins $\mathrm{KlaA}$ protein (ORF44) and KlaB/TelA (ORF45) were found. Therefore, the presence of this replicon in the cells may be beneficial in environments contaminated with this (mildly toxic) metalloid. This plasmid also contains several potential DNA repair proteins, including a UV protection protein (ORF26). ORFs of all the plasmids along with their potential function are listed in Supplementary Tables S2-S5.

The indicated phenotypic modules found within the O23A plasmids, and especially pO23A_p4, may provide the strain with particularly advantageous traits. As the three largest plasmids carry either MOB or TRA modules, they may actively participate in the dissemination of genes via horizontal gene transfer.

\section{Phage $\Phi 023 \mathrm{~A}$}

Another element of the Aeromonas sp. O23A mobilome is the prophage integrated within its chromosome, named $\Phi \mathrm{O} 23 \mathrm{~A}$. Following induction with mitomycin $\mathrm{C}$, phage particles were analyzed by TEM. TEM images showed that the virion has an icosahedral head approximately $65 \mathrm{~nm}$ in diameter and a tail approximately $100 \mathrm{~nm}$ long and $40 \mathrm{~nm}$ wide (Supplementary Figure S1). These morphological features indicate that the virus belongs to the Myoviridae family.

Sequence analysis showed that the genome of the phage ФO23A was 36847 bp DNA with a G+C content of $56.7 \%$, which is slightly lower than the average for the host genome (58.4\%). The comparison of the restriction profile of the $\Phi \mathrm{O} 23 \mathrm{~A}$ phage DNA (data not shown) with its nucleotide sequence yielded circular restriction maps of the phage genome, suggesting that the DNA molecules of $\Phi \mathrm{O} 23 \mathrm{~A}$ are linear and are circularly 
permuted. In the Aeromonas sp. O23A chromosome the $5^{\prime}$ end of the $\Phi \mathrm{O} 23 \mathrm{~A}$ prophage is preceded by a 112-bp direct repeat of an identical region present in the $\Phi \mathrm{O} 23 \mathrm{~A}$ genome (coordinates 36736 to 36847 ). This could be the attachment site to the host genome (attB). A part of this region corresponds to the $3^{\prime}$ end of tRNA-Leu gene, which in turn is the last of the three tRNA genes' cluster [tRNA-Gly(GCC), tRNA-Cys(GCA), tRNALeu(TAA)]. The phage $\Phi \mathrm{O} 23 \mathrm{~A}$ presumably utilizes chromosomal sequences encoding a tRNA gene for attachment, like many other temperate bacteriophages (e.g., HP1, ФLM21). In this case, integration of the phage $\Phi \mathrm{O} 23 \mathrm{~A}$ genome reconstitutes an intact copy of a leucine tRNA(TAA) gene because the 57-nucleotide terminal sequence (coordinates 36791 to 36847 ) of the ФO23A right arm is identical with the $3^{\prime}$ end of tRNA-Leu gene. The ФO23A genome containes 49 putative genes, which share a significant similarity at the protein level with the other sequences in GenBank. Putative functional assignments and significant similarities to the predicted genes are listed in Supplementary Table S6.

Comparison of the nucleotide sequence of $\Phi \mathrm{O} 23 \mathrm{~A}$ with the databank BlastN revealed a high degree of similarity (query coverage $65 \%$, $e$-value 0.0 ; coordinates $14262-22731$ ) with the P2-like ФO18P phage of A. media (DQ674738, Beilstein and Dreiseikelmann, 2008). Moreover, BLASTP analyses showed that the $\Phi \mathrm{O} 23 \mathrm{~A}$ proteome shares 36 homologs $(73.5 \%$ of ФО23A-encoded proteins) with $\Phi \mathrm{O} 18 \mathrm{P}$. The shared homologs are encoded in a collinear cluster of genes and include an integrase, transcription regulatory proteins, replication protein A, both terminases, portal and other structural proteins, holin and endolysin. Unique $\Phi \mathrm{O} 23 \mathrm{~A}$ proteins, not having their homologous counterparts in the $\Phi \mathrm{O} 18 \mathrm{P}$ genome are localized on the left and right ends of the $\Phi \mathrm{O} 23 \mathrm{~A}$ genome. These are: on the left arm ORF1 (putative transcriptional regulator), ORF3 (putative lysogenic conversion protein), ORF4 (CI repressor), ORF6 (Cox-like transcriptional regulator) and on the right arm: ORF46-47 (hypothetical proteins), ORF48 (HNH endonuclease), ORF49 (DNA-cytosine-methyltransferase). It is possible that ORF48 and ORF49 form together a restriction-modification system as similar pairs of homologous genes are found in other genomes (e.g., in Enterobacter hormaechei subsp. steigerwaltii strain 34998- AKZ84360 and AKZ84359).

The predicted protein product of the ORF49 gene showed similarity to C5-methylcytosine methytransferases (m5C MTases), including M.Eco4255I of Escherichia coli O69:H11 07-3763 and M.Eco3763II of E. coli O118:H16 07-4255, whose recognition sequence and target motif (GCCGGC, the methylated base is underlined) was identified in the genome by SMRT sequencing technology. The specificity of ORF49 was tested by comparative digestion of the pET-O23A_ФORF49 plasmid DNA, isolated from IPTG-induced and uninduced E. coli cultures, with PdiI (GCCGGC), BsuRI (GGCC), Bsh1236I (CGCG), and Hin6I (GCGC) restriction enzymes. The DNA of pET-O23A_ФORF49 isolated from the induced culture was cleaved by all the tested restriction enzymes, with the exception of PdiI. In contrast, the pET-O23A_ФORF49, DNA isolated from the non-induced culture was susceptible to all restriction enzymes, including PdiI. The DNAs of $\Phi \mathrm{O} 23 \mathrm{~A}$, with
31 GCCGGC was completely resistant to PdiI digestion (data not shown). Furthermore, chromosomal DNA of Aeromonas sp. O23A was also completely resistant to PdiI digestion indicting that ORF49 is expressed during $\Phi \mathrm{O} 23 \mathrm{~A}$ lysogeny. It is possible that ORF48-encoded putative $\mathrm{HNH}$ nuclease is also active during the temperate state of the $\Phi \mathrm{O} 23 \mathrm{~A}$ phage and together they constitute protection against infection of their host by other phages or foreign DNA.

\section{Other Mobile Elements}

Apart from 4 plasmids and a prophage, the mobilome of Aeromonas sp. O23A comprises a number of putative transposable elements. In total 22 genes encoding transposases of transposable elements were identified. According to the ISfinder database (Siguier et al., 2006), they belong to 8 families, namely: IS 110 (IS 1111 group), IS1595 (IS1595 group), IS200/IS605 (IS200 group), IS3 (IS3 and IS407 groups), IS481, IS5 (IS5 and IS903 groups), IS630, and Tn3. All the transposable elements were distinguished exclusively in the chromosome.

\section{CRISPR System}

Although gene exchange between the bacterial strains is often beneficial, excessive DNA transfer may cause damage to the cells. One of the common defense mechanisms against foreign DNA are elements of the CRISPR system. Within the genome of Aeromonas sp. O23A a CRISPR locus containing six genes encoding CRISPR-associated proteins and the upstream region containing 54 short spacer sequences ( 53 of 32 bp in length and 1 of $33 \mathrm{bp}$ ) was identified. BLASTN comparative analysis revealed that some of the spacers show sequence similarity to: (i) phages, including Aeromonas phage vB_AsaM-56 (Acc no. JQ177063), Aeromonas phage DH1 clone SEQDH5 (Acc. no. EU515217) and Sinorhizobium meliloti phage PBC5 (Acc. no. AF448724); (ii) plasmids, including Ralstonia solanacearum PSI07 megaplasmid mpPSI07 (Acc no. FP885891), Azospirillum brasilense Sp7 plasmid ABSP7_p3 (Acc. no. CP012917), Ensifer adhaerens OV14 plasmid pOV14b (CP007239.1), Streptomyces clavuligerus F613-1 plasmid pSCL4 (Acc. no. CP016560), Burkholderia rhizoxinica HKI 454 plasmid pBRH01 (Acc. no. FR687360), Pseudomonas fluorescens SBW25 plasmid pQBR103 (Acc. no. AM235768), Nitrobacter hamburgensis X14 plasmid 1 (Acc. no. CP000320), Sphingomonas sp. NIC1 plasmid unnamed2 (Acc. no. CP015523) and Rhizobium etli bv. mimosae strain Mim1 plasmid pRetMIM1e (Acc. no. CP005955); and (iii) genomic islands, including A. salmonicida subsp. salmonicida JF3224 genomic island AsaGEI2b (Acc. no. KP861348) and A. salmonicida HER1085 genomic island AsaGEI1b (Acc. no. KJ626179).

\section{Heavy Metal Resistance and Metabolism Genes Within the Aeromonas sp. 023A Genome}

\section{Arsenic Metabolism and Resistance}

Bioinformatic analysis of the chromosome sequence of $\mathrm{O} 23 \mathrm{~A}$ indicated the presence of two genetic modules related to arsenic resistance and metabolism. The first one (Module 1) consisting of a set of ars and arr genes conferring arsenic resistance and 


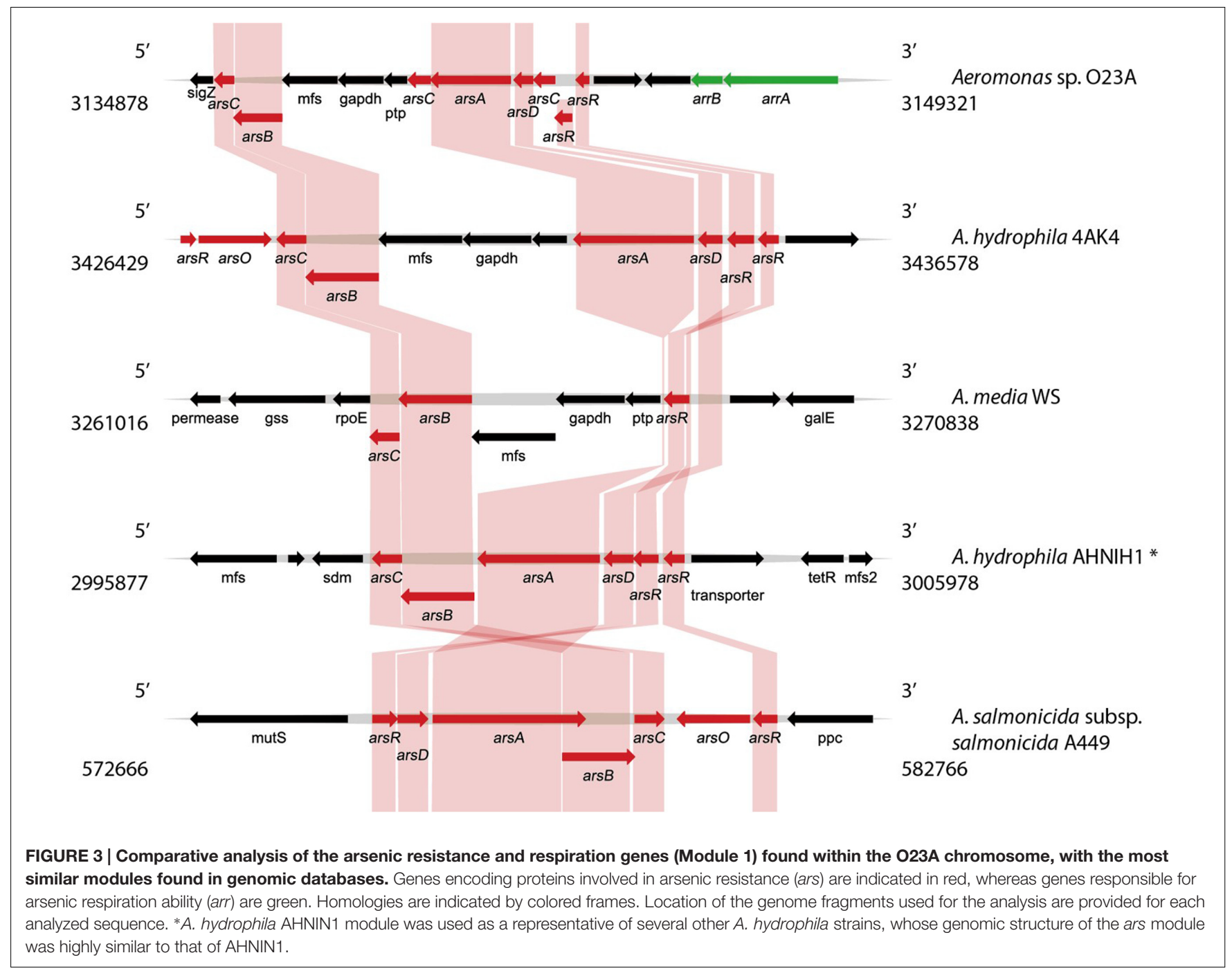

respiration abilities, respectively, is located between 3134878 and $3149321 \mathrm{nt}$ of the chromosome (Figure 3). The second arsenic resistance module (Module 2) encoded by the O23A chromosome is located between 3266814 and $3278484 \mathrm{nt}$ (Figure 4). It consists of a complete set of ars genes, typical for arsenic resistance operon (arsRDABC) found in many strains, including E. coli (Carlin et al., 1995; Edmundson and Horsfall, 2015), but lacks genes for arsenic respiration. Moreover, it differs from the recognized model arsenic resistance module of Shewanella ANA3. On the other hand, the arr module of $\mathrm{O} 23 \mathrm{~A}$ more closely resembles that of ANA3, which comprises $\operatorname{arr} A$ and $\operatorname{arrB}$ genes, whose products (ArrA and ArrB proteins, respectively) are both required for respiratory $\mathrm{As}(\mathrm{V})$ reduction (Saltikov et al., 2005), especially when arsenate concentrations are low (Malasarn et al., 2004). The potential protein product of the $\operatorname{arrB}$ gene of O23A is in $60 \%$ identical with ArrB protein (AAQ01673.1) of ANA3. The similarity of the putative ArrA protein of $\mathrm{O} 23 \mathrm{~A}$ to that of ANA3 is not as high. Nevertheless, the functionality of the arr module of $\mathrm{O} 23 \mathrm{~A}$ has been confirmed in the previous studies, where O23A, along with other Aeromonas spp. strains isolated from the Zloty Stok mine (Drewniak et al., 2010) was found to dissimilatorily reduce arsenate to arsenite in a respiratory process (Uhrynowski et al., 2017).

The differences in the structure of the Modules 1 and 2 suggest that they are of distinct origin. The ars genes in Module 1 do not form a concise operon like in Module 2 , but are separated from each other by genes encoding predicted: (i) permease of the major facilitator superfamily $(m f s)$, (ii) NAD-dependent glyceraldehyde-3-phosphate dehydrogenase (EC 1.2.1.12, gapdh), and (iii) protein-tyrosine phosphatase ( $p t p$ ). Interestingly, Module 1 comprises two copies of the arsR gene and three copies of the ars $C$ gene, which may indicate recent rearrangements within the $\mathrm{O} 23 \mathrm{~A}$ chromosome, potentially based on homologous recombination. Moreover, the presence of $m f s$, gapdh, and ptp genes between the ars $A$ and ars $B$ genes, also seems to be an insertion. Nevertheless, homology of both the O23A arsenic resistance modules with other Aeromonas strains was found.

Comparative analysis of Module 1 with other, most identical gene modules involved in arsenic transformations found in 


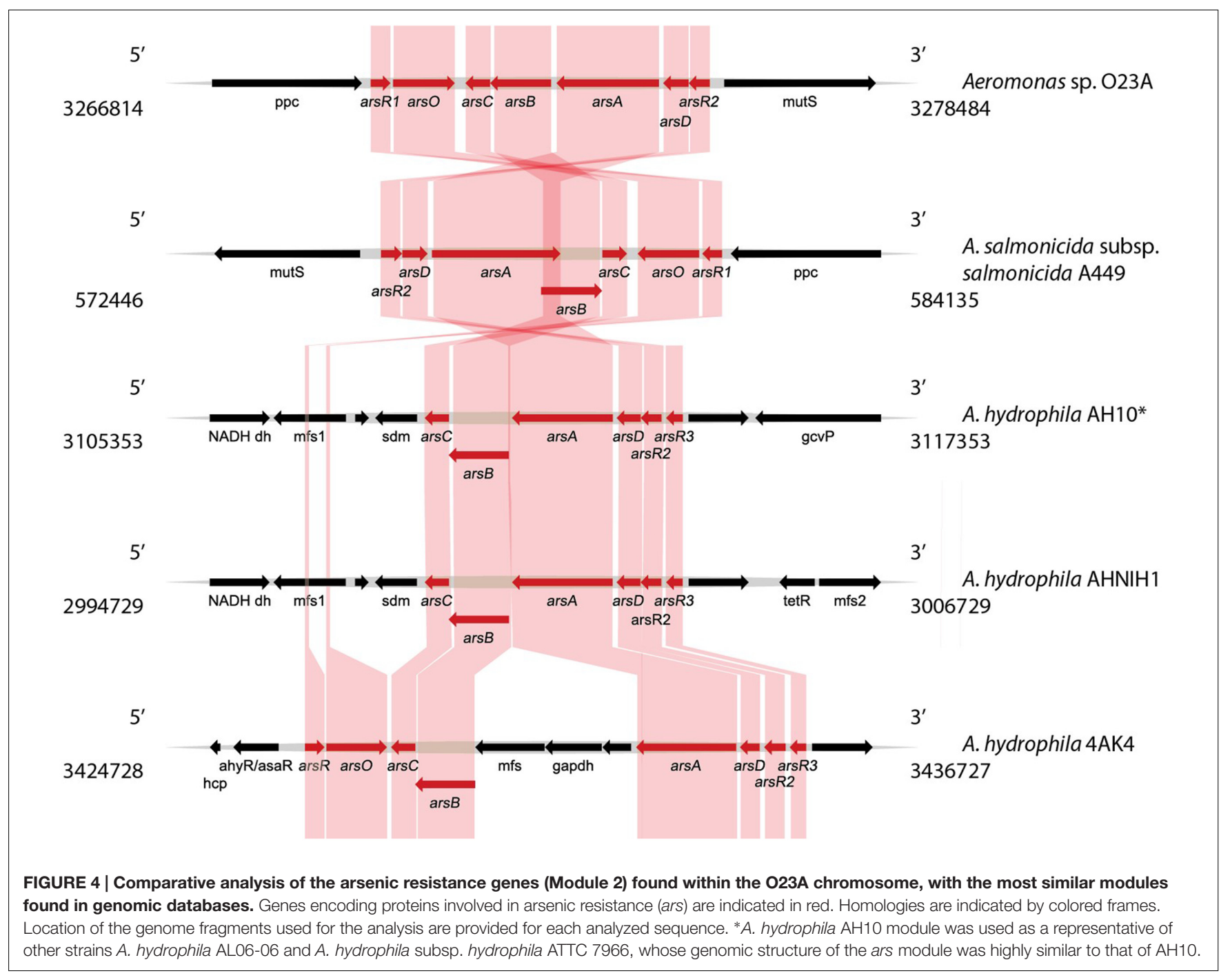

the genomic databases using BLAST is shown in Figure 3. Although the analyses indicted similarity of this module to other arsenic modules found in Aeromonas spp. genomes, this is only with regard to the ars part of the O23A module. No significant similarities covering both the potential arr genes have been found, making the O23A module unique among other Aeromonas species. The arsenic resistance part of Module 1 of O23A most closely resembles that of A. hydrophila 4AK4, as in both the strains, ars $A$ and $\operatorname{ars} B$ genes are separated by $m f s$, gapdh, and potentially $p t p$ genes. Both the modules also show limited similarity to the partial ars module of A. media WS, which also includes the $m f s$, gapdh, and $p t p$ insertion, but lacks the ars $A$ gene in close proximity to the other ars genes.

In turn, Module 2 of O23A appears to be most closely related to that of $A$. salmonicida subsp. salmonicida A449, even though their orientation in respective genomes is different (Figure 4). This again suggests a close relationship of $\mathrm{O} 23 \mathrm{~A}$ with A449. The modules of both the strains include the ars $O$ gene encoding a putative monooxygenase, which is not present in several other homologous modules found in several strains of A. hydrophila. An exception is the ars module of $A$. hydrophila $4 \mathrm{AK} 4$, which comprises the ars $\mathrm{O}$ gene. Interestingly, the 4AK4 module shows homology with both ars modules of O23A.

Though differing in orientation, the ars modules of A. hydrophila AH10 and AL06-06, as well as A. hydrophila subsp. hydrophila ATTC 7966, have almost identical structure. However, the location of additional copies of the ars $R$ gene varies from that of $\mathrm{O} 23 \mathrm{~A}$ and $\mathrm{A} 449$, as in the former both arsR gene copies are localized together, whereas in the latter two strains, these genes constitute the boundaries of the module, and they are oppositely oriented. Therefore, the module of $4 \mathrm{AK} 4$ seems to be a combination of both types of resistance modules - that of O23A/A449 and those of other A. hydrophila strains.

Interestingly, further analysis of the O23A genome revealed another (partial) copy of the $\operatorname{arr} A$ gene within the chromosome of O23A. It is localized between 2790088-2790975 nt, and in close proximity to this location (upstream and downstream), 
several proteins possibly involved in the horizontal gene transfer are found (mobile element proteins: 2780913-2779794 nt, 2795165-2795716 nt). The potential protein product of this arrA gene copy shows resemblance to A. salmonicida subsp. pectinolytica $34 \mathrm{mel}$. Comparative analyses revealed that two potential proteins encoded within the $34 \mathrm{mel}$ genome may encode ArrB homologs, but their identity with the model respiratory arsenate reductase is low (27-31\%). Therefore, it cannot be unambiguously concluded that the $34 \mathrm{mel}$ strain is capable of dissimilatory arsenate reduction without physiological studies. Nevertheless, this suggests the potential capabilities of the clinical strains Aeromonas spp. to transform arsenic, which yet has to be revealed by in silico screening, followed by thorough in vitro studies. This result may also indicate that the dissimilatory arsenate reduction ability may either have been lost during genome reduction in pathogenic strains such as A. salmonicida, or acquired by $\mathrm{O} 23 \mathrm{~A}$ from other DARB found within the Zloty Stok mine, e.g., from Shewanella sp. O23S (Drewniak et al., 2015) through horizontal gene transfer. However, the latter hypothesis may only be confirmed when the genomes of other DARB from the mine are sequenced.

In the case of $\mathrm{O} 23 \mathrm{~A}$, the functionality of the ars modules was confirmed by remarkably high resistance to both inorganic arsenic forms As(V) $325 \mathrm{mM}$ and As(III) $10 \mathrm{mM}$, as indicated in the previous study (Uhrynowski et al., 2017). This was expected, as the strain was isolated from an environment highly contaminated with arsenic. This is also consistent with the presence of two potentially functional arsenic resistance modules, whose protein products facilitate subsequent reduction (ArsC) and active transport (ArsA/ArsB) of arsenate out of the cell.

\section{Resistance to Other Heavy Metals}

The Aeromonas sp. O23A proteome was mapped against the BacMet database (Pal et al., 2014). This allowed for the identification of 98 genes possibly involved in resistance to inorganic ions. Each gene in this pool was manually verified and after deletion of the false positive hits, 15 heavy metal resistance $(h m r)$ genetic modules were distinguished, including: (i-ii) O23A_p1143 and O23A_p4209 genes encoding magnesium/cobalt efflux protein CorA, (iii) O23A_p0410 encoding copper homeostasis protein CutC, (iv-vi) O23A_p1375, O23A_p3014 and O23A_p3780 encoding copper-translocating P-type ATPase (which may be involved in copper, lead, cadmium, zinc and mercury transport), (vii-viii) O23A_p2248-2249 and O23A_p2630-2631 encoding cobalt, zinc and cadmium resistance systems (CzcAB), (ix) O23A_p2639-2640 encoding copper resistance system CopAB, (x) O23A_p2978 encoding magnesium and cobalt efflux protein CorB, (xi) O23A_p3017 encoding cobalt, zinc and cadmium resistance protein $\mathrm{CzcD}$, (xii-xiii) O23A_p3309 and O23A_p3510 encoding divalent metal cations (Fe/Co/Zn/Cd) transporters of FieF family.

The functionality of the hmr modules found within the O23A genome was assessed by MIC analyses. It was found that apart from high concentrations of As(III) and As(V), the strain shows resistance to at least $1 \mathrm{mM}$ of $\mathrm{Cd}(\mathrm{II})$ and $\mathrm{Co}(\mathrm{II})$, and - as it was previously observed - to $1 \mathrm{mM}$ of and $\mathrm{Zn}(\mathrm{II}), 2 \mathrm{mM}$ of $\mathrm{Cu}(\mathrm{II})$ and $5 \mathrm{mM}$ of $\mathrm{Ni}$ (II) (Uhrynowski et al., 2017). This indicates that at least one copy of the aforementioned resistance genes encodes a functional product. However, the strain was found to be incapable of growth in the presence of $1 \mathrm{mM}$ of $\mathrm{Cr}(\mathrm{VI})$ or $0.1 \mathrm{mM}$ of $\mathrm{Hg}$ (II). This may suggest that the copper-translocating P-type ATPases are not functional, or their affinity toward mercury is limited. This is in contrast to other Aeromonas spp., e.g., A. hydrophila KT20, which is not only resistant, but is directly involved in $\mathrm{Hg}$ (II) reduction (Watts and Lloyd, 2013).

\section{Siderophore Production and Mineral Dissolution}

The use of antiSMASH tool (Weber et al., 2015) enabled the identification of two DNA regions within the chromosome of Aeromonas sp. O23A, which contain gene clusters possibly responsible for the synthesis of non-ribosomal peptides (putative siderophores). The first gene cluster (coordinates 1,155,068$1,209,363$ ) encoding non-ribosomal peptide synthetase is most probably responsible for the synthesis of an enterobactin-like siderophore, as $20 \%$ of its genes shows similarity to enterobactin biosynthetic gene cluster identified in Enterobacteriaceae. Another gene cluster (coordinates 3,834,873-3,882,845) found within the $\mathrm{O} 23 \mathrm{~A}$ chromosome is composed of genes, of which $90 \%$ show similarity the pseudomonine biosynthetic gene cluster. Pseudomonine is an isoxazolidone with siderophoric activity, which was isolated from Pseudomonas fluorescens AH2 (Anthoni et al., 1995).

The O23A strain was previously found to be capable of arsenic mineral dissolution (Drewniak et al., 2010) and hydroxamate siderophore production (Uhrynowski et al., 2017). However, the actual in vitro activity of the produced siderophores has not been tested. Cultivation of the strain on GASN medium, which lacks iron ions, stimulates bacteria to produce siderophores. Mineral dissolution experiments using filtered culture supernatants showed that after 7 days of incubation, concentration of the dissolved iron ions increased by approx. $2.0 \mathrm{mg} / \mathrm{l}$ and $3.0 \mathrm{mg} / \mathrm{l}$ for the experiments with arsenopyrite and pyrite, respectively, compared to the control (sterile GASN medium). Therefore, if suitable conditions are provided, the strain can contribute to the extraction and controlled release of heavy metals from mine deposits (solid waste) and therefore bioremediation, through the reduction of their environmental load.

On the other hand, siderophore production ability is regarded as typical for pathogens. By facilitating iron uptake, siderophores enable the growth of the strains during infections, when natural defensive mechanisms of the infected host cause iron ions to become less available to microorganisms, e.g., removed from the bloodstream (Parrow et al., 2013). This, in the light of the aforementioned similarities with the fish pathogen $A$. salmonicida, suggests that $\mathrm{O} 23 \mathrm{~A}$ genome encodes proteins, which may be involved in pathogenesis. Therefore, the application potential of the strain may be limited to closed bioremediation systems, where appropriate safety measures are taken before the remediated soil/water is released back into the environment. 


\section{CONCLUSION}

Aeromonads are known for their ubiquitous nature, which is to a great extent determined by the carried pool of genes, and especially those found within mobile genetic elements. Genomic research is mostly focused on clinically important determinants of these bacteria, such as antibiotic resistance genes, and much less attention is being paid to other adaptive genes, related to, e.g., heavy metal resistance. Such capabilities often remain undiscovered, even though they may affect the entire biology of the microorganisms, as well as the surrounding environment. This is unfortunate, as the determination of the gene pool of microorganisms can provide particularly valuable and interesting information about their abilities, also those which are not demonstrated during growth under optimum and/or laboratory conditions.

In this work, Aeromonas sp. O23A was subjected to in silico analyses, the results of which were verified based on in vitro studies. Genomic analysis enabled screening for traits of potential significance in arsenic metabolism, while physiological tests allowed to experimentally confirm the functionality of the identified genes and phenotypic modules. Genetic modules coding for arsenic resistance and metabolism have been found, and compared with other closely related strains.

The performed comparative analyses suggest that the complete set of genes for arsenic respiration found in $\mathrm{O} 23 \mathrm{~A}$ is unique. No other similar arr gene modules were found in Aeromonas spp. strains, whose genomes had been sequenced. However, it cannot be ruled out, that other Aeromonads may be capable of dissimilatory arsenate reduction, especially as genes encoding potential proteins homologous to ArrA and ArrB were distinguished within other Aeromonas spp. The functionality of the indicated proteins has yet to be experimentally determined.

The combined genetic-physiological approach seems to be the best way to screen for and confirm the potential of not only Aeromonads, but also other strains. Based on such analysis, $\mathrm{O} 23 \mathrm{~A}$ was found to be resistant to several heavy metal ions, and capable of producing siderophores, what indicates that the strain carries genes coding for potentially desirable, and undesirable traits, depending on its application. Due to arsenic transformation abilities, O23A has a great application potential in bioremediation, which may be hindered only by the potential

\section{REFERENCES}

Adamczuk, M., and Dziewit, L. (2016). Genome-based insights into the resistome and mobilome of multidrug-resistant Aeromonas sp. ARM81 isolated from wastewater. Arch. Microbiol. 199, 177-183. doi: 10.1007/s00203-016-1285-6

Altschul, S. F., Madden, T. L., Schäffer, A. A., Zhang, J., Zhang, Z., Miller, W., et al. (1997). Gapped BLAST and PSI-BLAST: a new generation of protein database search programs. Nucleic Acids Res. 25, 3389-3402. doi: 10.1093/nar/25.17.3389

Anthoni, U., Christophersen, C., Nielsen, P. H., Gram, L., and Petersen, B. O. (1995). Pseudomonine, an isoxazolidone with siderophoric activity from pseudomonas fluorescens AH2 isolated from Lake Victorian Nile Perch. J. Nat. Prod. 58, 1786-1789. doi: 10.1021/np50125a026

Aziz, R. K., Bartels, D., Best, A. A., DeJongh, M., Disz, T., Edwards, R. A., et al. (2008). The RAST server: rapid annotations using subsystems technology. BMC Genomics 9:75. doi: 10.1186/1471-2164-9-75 pathogenicity of the strain, which will be assessed in further studies.

\section{AUTHOR CONTRIBUTIONS}

WU participated in genome assembly and its deposition in the GenBank database, performed bioinformatic analyses of heavy metal resistance and metabolism genes, annotated the plasmids, carried out the physiological experiments, analyzed the data and wrote the manuscript. $\mathrm{PD}$ and $\mathrm{LDz}$ performed a general analysis of the O23A genome, including the investigation of metabolic pathways and mobile genetic elements. MR performed a complete phage analysis and was involved in article preparation. PK, LL, and DA were involved in DNA sequencing, assembly and genome annotation. LDr is the project manager, and was involved in data consultation. All authors read and approved the final manuscript.

\section{FUNDING}

This work was supported by the Polish Ministry of Science and Higher Education in the form of a grant Iuventus Plus no. $0107 / \mathrm{IP} 1 / 2015 / 73$. Part of this work, i.e., sequencing and genome assembly, was also supported by the EU European Regional Development Fund, the Operational Program Innovative Economy 2007-2013, the project support Agreement POIG.01.01.02-14- 054/09-00.

\section{ACKNOWLEDGMENT}

The authors would like to thank Mr. Jan Gawor for his help in genome sequencing and assembly.

\section{SUPPLEMENTARY MATERIAL}

The Supplementary Material for this article can be found online at: http://journal.frontiersin.org/article/10.3389/fmicb. 2017.00936/full\#supplementary-material

Beilstein, F., and Dreiseikelmann, B. (2008). Temperate bacteriophage $\phi \mathrm{O} 18 \mathrm{P}$ from an Aeromonas media isolate: characterization and complete genome sequence. Virology 30, 25-29. doi: 10.1016/j.virol.2007. 11.016

Bultreys, A., and Gheysen, I. (2000). Production and comparison of peptide siderophores from strains of distantly related pathovars of Pseudomonas syringae and Pseudomonas viridiflava LMG 2352. Appl. Environ. Microbiol. 66, 325-331. doi: 10.1128/AEM.66.1.325-331.2000

Carlin, A., Shi, W., Dey, S., and Rosen, B. P. (1995). The ars operon of Escherichia coli confers arsenical and antimonial resistance. J. Bacteriol. 177, 981-986.

Carver, T., Berriman, M., Tivey, A., Patel, C., Böhme, U., Barrell, B. G., et al. (2008). Artemis and ACT: viewing, annotating and comparing sequences stored in a relational database. Bioinformatics 24, 2672-2676. doi: 10.1093/bioinformatics/ btn529 
Chen, P. L., Lamy, B., and Ko, W. C. (2016). Aeromonas dhakensis, an increasingly recognized human pathogen. Front. Microbiol. 7:793. doi: 10.3389/fmicb.2016. 00793

Darmon, E., and Leach, D. R. F. (2014). Bacterial genome instability. Microbiol. Mol. Biol. Rev. 78, 1-39. doi: 10.1128/MMBR.00035-13

Drewniak, L., Krawczyk, P. S., Mielnicki, S., Adamska, D., Sobczak, A., Lipinski, L., et al. (2016). Physiological and metagenomic analyses of microbial mats involved in self-purification of mine waters contaminated with heavy metals. Front. Microbiol. 7:1252. doi: 10.3389/fmicb.2016.01252

Drewniak, L., Maryan, N., Lewandowski, W., Kaczanowski, S., and Sklodowska, A. (2012). The contribution of microbial mats to the arsenic geochemistry of an ancient gold mine. Environ. Pollut. 162, 190-201. doi: 10.1016/j.envpol.2011. 11.023

Drewniak, L., Matlakowska, R., Rewerski, B., and Sklodowska, A. (2010). Arsenic release from gold mine rocks mediated by the activity of indigenous bacteria. Hydrometallurgy 104, 437-442. doi: 10.1016/j.hydromet.2010. 02.025

Drewniak, L., Rajpert, L., Mantur, A., and Sklodowska, A. (2014). Dissolution of arsenic minerals mediated by dissimilatory arsenate reducing bacteria: estimation of the physiological potential for arsenic mobilization. Biomed. Res. Int. 2014:841892. doi: 10.1155/2014/841892

Drewniak, L., Stasiuk, R., Uhrynowski, W., and Sklodowska, A. (2015). Shewanella sp. O23S as a driving agent of a system utilizing dissimilatory arsenate-reducing bacteria responsible for self-cleaning of water contaminated with arsenic. Int. J. Mol. Sci. 16, 14409-14427. doi: 10.3390/ijms160714409

Du, H., Pang, M., Dong, Y., Wu, Y., Wang, N., Liu, J., et al. (2016). Identification and characterization of an Aeromonas hydrophila oligopeptidase gene pepF negatively related to biofilm formation. Front. Microbiol. 7:1497. doi: 10.3389/ fmicb.2016.01497

Dumontet, S., Krovacek, K., Svenson, S. B., Pasquale, V., Baloda, S. B., and Figliuolo, G. (2000). Prevalence and diversity of Aeromonas and Vibrio spp. in coastal waters of Southern Italy. Comp. Immunol. Microbiol. Infect. Dis. 23, 53-72. doi: 10.1016/S0147-9571(99)00059-4

Dziewit, L., and Bartosik, D. (2014). Plasmids of psychrophilic and psychrotolerant bacteria and their role in adaptation to cold environments. Front. Microbiol. 5:596. doi: 10.3389/fmicb.2014.00596

Dziewit, L., Cegielski, A., Romaniuk, K., Uhrynowski, W., Szych, A., Niesiobedzki, P., et al. (2013). Plasmid diversity in arctic strains of Psychrobacter spp. Extremophiles 17, 433-444. doi: 10.1007/s00792-013-0521-0

Edmundson, M. C., and Horsfall, L. (2015). Construction of a modular arsenicresistance operon in E. coli and the production of arsenic nanoparticles. Front. Bioeng. Biotechnol. 3:160. doi: 10.3389/fbioe.2015.00160

Felsenstein, J. (1985). Confidence limits on phylogenies: an approach using the bootstrap. Evolution 39, 783-791.

Karp, P. D., Latendresse, M., and Caspi, R. (2011). The pathway tools pathway prediction algorithm. Stand. Genomic Sci. 5, 424-429. doi: 10.4056/sigs.1794338

Kimura, M. (1980). A simple method for estimating evolutionary rates of base substitutions through comparative studies of nucleotide sequences. J. Mol. Evol. $16,111-120$.

Kumar, S., Stecher, G., Tamura, K., and Medicine, E. (2016). MEGA7: molecular evolutionary genetics analysis version 7.0 for bigger datasets. Mol. Biol. Evol. 33, 1870-1874. doi: 10.1093/molbev/msw054

Malasarn, D., Saltikov, C. W., Campbell, K. M., Santini, J. M., Hering, J. G., and Newman, D. K. (2004). arrA is a reliable marker for As(V) respiration. Science 306, 455-455. doi: 10.1126/science.1102374

Nishida, H. (2013). Genome DNA sequence variation, evolution, and function in bacteria and archaea. Curr. Issues Mol. Biol. 15, 19-24.

Ogugbue, C. J., and Sawidis, T. (2011). Bioremediation and detoxification of synthetic wastewater containing triarylmethane dyes by Aeromonas hydrophila isolated from industrial effluent. Biotechnol. Res. Int. 2011:967925. doi: 10.4061/ $2011 / 967925$
Okibe, N., Koga, M., Morishita, S., Tanaka, M., Heguri, S., Asano, S., et al. (2014). Microbial formation of crystalline scorodite for treatment of As(III)-bearing copper refinery process solution using Acidianus brierleyi. Hydrometallurgy 143, 34-41. doi: 10.1016/j.hydromet.2014.01.008

Pal, C., Bengtsson-Palme, J., Rensing, C., Kristiansson, E., and Larsson, D. G. J. (2014). BacMet: antibacterial biocide and metal resistance genes database. Nucleic Acids Res. 42, 737-743. doi: 10.1093/nar/gkt1252

Parrow, N. L., Fleming, R. E., and Minnick, M. F. (2013). Sequestration and scavenging of iron in infection. Infect. Immun. 81, 3503-3514. doi: 10.1128/IAI. 00602-13

Rasmussen-Ivey, C. R., Figueras, M. J., McGarey, D., and Liles, M. R. (2016). Virulence factors of Aeromonas hydrophila: in the wake of reclassification. Front. Microbiol. 7:1337. doi: 10.3389/fmicb.2016.01337

Roberts, R. J., Vincze, T., Posfai, J., and Macelis, D. (2015). REBASE-a database for DNA restriction and modification: enzymes, genes and genomes. Nucleic Acids Res. 43, D298-D299. doi: 10.1093/nar/gku1046

Saitou, N., and Nei, M. (1987). The neighbor-joining method: a new method for reconstructing phylogenetic trees. Mol. Biol. Evol. 4, 406-425.

Saltikov, C., Wildman, R. Jr., and Newman, D. K. (2005). Expression dynamics of arsenic respiration and detoxification in Shewanella sp. Strain ANA-3. J. Bacteriol. 187, 7390-7396. doi: 10.1128/JB.187.21.7390

Sambrook, J., and Russell, D. W. (2001). Molecular Cloning: A Laboratory Manual. Cold Spring Harbor, NY: Cold Spring Harbor Laboratory Press.

Siguier, P., Perochon, J., Lestrade, L., Mahillon, J., and Chandler, M. (2006). ISfinder: the reference centre for bacterial insertion sequences. Nucleic Acids Res. 34, D32-D36. doi: 10.1093/nar/gkj014

Teunis, P., and Figueras, M. J. (2016). Reassessment of the enteropathogenicity of Mesophilic aeromonas species. Front. Microbiol. 7:1395. doi: 10.3389/fmicb. 2016.01395

Tomás, J. M. (2012). The main Aeromonas pathogenic factors. ISRN Microbiol. 2012:256261. doi: 10.5402/2012/256261

Uhrynowski, W., Debiec, K., Sklodowska, A., and Drewniak, L. (2017). The role of dissimilatory arsenate reducing bacteria in the biogeochemical cycle of arsenic based on the physiological and functional analysis of Aeromonas sp. O23A. Sci. Total Environ. 598, 680-689. doi: 10.1016/j.scitotenv.2017.04.137

Wang, X.-J., Chen, X.-P., Kappler, A., Sun, G.-X., and Zhu, Y.-G. (2009). Arsenic binding to iron (II) minerals produced by an iron (III)-reducing aeromonas strain isolated from paddy soil. Environ. Toxicol. Chem. 28, 2255-2262. doi: 10.1897/09-085.1

Watts, M. P., and Lloyd, J. R. (2013). "Bioremediation via microbial metal reduction," in Microbial Metal Respiration: From Geochemistry to Potential Applications, eds J. Gescher and A. Kappler (Berlin: Springer), 161-201. doi: 10.1007/978-3-642-32867-1

Weber, T., Blin, K., Duddela, S., Krug, D., Kim, H. U., Bruccoleri, R., et al. (2015). AntiSMASH 3.0-A comprehensive resource for the genome mining of biosynthetic gene clusters. Nucleic Acids Res. 43, W237-W243. doi: 10.1093/ nar/gkv437

Zhou, Y., Liang, Y., Lynch, K. H., Dennis, J. J., and Wishart, D. S. (2011). PHAST: a fast phage search tool. Nucleic Acids Res. 39, 347-352. doi: 10.1093/nar/gkr485

Conflict of Interest Statement: The authors declare that the research was conducted in the absence of any commercial or financial relationships that could be construed as a potential conflict of interest.

Copyright (c) 2017 Uhrynowski, Decewicz, Dziewit, Radlinska, Krawczyk, Lipinski, Adamska and Drewniak. This is an open-access article distributed under the terms of the Creative Commons Attribution License (CC BY). The use, distribution or reproduction in other forums is permitted, provided the original author(s) or licensor are credited and that the original publication in this journal is cited, in accordance with accepted academic practice. No use, distribution or reproduction is permitted which does not comply with these terms. 